\title{
AN APPROXIMATION FOR THE INVERSE FIRST PASSAGE TIME PROBLEM
}

\author{
JING-SHENG SONG, ${ }^{*}$ AND \\ PAUL ZIPKIN, ${ }^{* * *}$ Duke University
}

\begin{abstract}
We propose an approximation for the inverse first passage time problem. It is similar in spirit and method to the tangent approximation for the original first passage time problem. We provide evidence that the technique is quite accurate in many cases. We also identify some cases where the approximation performs poorly.
\end{abstract}

Keywords: Inverse first passage time; Wiener process; approximation

2010 Mathematics Subject Classification: Primary 60J65

Secondary $65 \mathrm{~N} 21 ; 58 \mathrm{~J} 35$

\section{Introduction}

The first passage time of a stochastic process $W=\{W(t): t \geq 0\}$ to a boundary $b=b(t)$ is

$$
\tau=\inf \{t>0: W(t) \geq b(t)\} .
$$

The first passage time (FPT) problem is to find the distribution $F$ of $\tau$, given $b$. Here we are mainly concerned with the inverse first passage time (IFPT) problem: given $F$, find the corresponding $b$. We focus on the case where $W$ is a standard Wiener process starting at $W(0)=0$. We assume that $F$ is continuous, with a continuous, strictly positive density $f$.

The IFPT problem has applications in biology, finance, and other areas. Recently, Song and Zipkin [12] formulated an inventory-control problem which, in certain cases, turns out to be equivalent to the IFPT problem. See the other references below for further discussion of applications and pointers to the literature on them.

The IFPT problem has received considerable attention recently. Cheng et al. [2] showed that the problem is well posed-given $F$, there exists a unique solution (in the weak or viscosity sense). Zucca and Sacerdote [14] described two solution methods, one employing a piecewiselinear approximation of the boundary, and the other based on discretizing a Volterra integral equation. Song and Zipkin [12] presented a different algorithmic approach. Other work on this and related problems include [1], [7], and [10].

We propose a simple approximation for the IFPT problem. It is similar in spirit and method to the tangent approximation for the original FPT problem. We provide evidence that the technique is quite accurate in many cases, with errors of just a few percent. Of course, it is no substitute for the exact methods mentioned above.

We also identify some problematic cases, where the approximation performs poorly. These have strongly bimodal or oscillating densities $f$. We suspect that such cases may prove challenging for other methods for both the FPT and the IFPT problems.

Received 11 May 2010; revision received 14 October 2010.

* Postal address: Fuqua School of Business, Duke University, Durham, NC, USA.

** Email address: paul.zipkin@duke.edu 
The tangent approximation for the FPT problem, originally proposed in [3] and [13], approximates $b$ locally by a straight line. It has been studied intensively by Lerche [8], among others. Several refinements have been suggested, including the hazard-rate tangent approximation of Roberts and Shortland [11]. Our method can be viewed as a natural inverse of this approach.

\section{Method}

If $b(t)$ is the linear function $\alpha+\beta t$ with $\alpha>0$ and any real $\beta$, then $\tau$ has an inverse Gaussian (or Wald, or Bachelier-Lévy) distribution:

$$
\begin{aligned}
& f_{(\alpha, \beta)}(t)=\frac{\alpha}{t^{3 / 2}} \phi\left(\frac{\alpha+\beta t}{\sqrt{t}}\right), \\
& F_{(\alpha, \beta)}(t)=\exp (-2 \alpha \beta) \bar{\Phi}\left(\frac{\alpha-\beta t}{\sqrt{t}}\right)+\bar{\Phi}\left(\frac{\alpha+\beta t}{\sqrt{t}}\right) .
\end{aligned}
$$

Here, $\phi$ is the standard normal density function, $\Phi$ is the corresponding cumulative distribution function, and $\bar{\Phi}=1-\Phi$. This is one of the few cases where the exact solutions to the FPT and the IFPT problems are known.

Note that the density $f_{(\alpha, \beta)}$ is always unimodal. Also, the qualitative behavior of $F_{(\alpha, \beta)}$ depends on the sign of $\beta$.

1. When $\beta<0, F_{(\alpha, \beta)}$ is a proper or nondefective distribution (i.e. $F_{(\alpha, \beta)}(\infty)=1$ ) with finite moments of all orders.

2. When $\beta=0, F_{(\alpha, \beta)}$ is still proper, but its mean is infinite.

3. When $\beta>0, F_{(\alpha, \beta)}$ is improper or defective, with $F_{(\alpha, \beta)}(\infty)=\exp (-2 \alpha \beta)$.

This distribution can be expressed in another way. Define

$$
z=\frac{\alpha+\beta t}{\sqrt{t}}, \quad y=\frac{\alpha-\beta t}{\sqrt{t}} .
$$

Then, $\alpha=\sqrt{t}(z+y) / 2$ and $\alpha \beta=\left(z^{2}-y^{2}\right) / 4$. So,

$$
\begin{aligned}
& f_{(\alpha, \beta)}(t)=\frac{z+y}{2 t} \phi(z), \\
& F_{(\alpha, \beta)}(t)=\exp \left(-\frac{z^{2}-y^{2}}{2}\right) \bar{\Phi}(y)+\bar{\Phi}(z)=\frac{\phi(z)}{\phi(y)} \bar{\Phi}(y)+\bar{\Phi}(z) .
\end{aligned}
$$

For the case where $F$ is not inverse Gaussian, we propose the following method. For each $t$, fit $\alpha$ and $\beta$ to the given values of $F(t)$ and $f(t)$ using the formulae above. Call the results $\hat{\alpha}=\hat{\alpha}(t)$ and $\hat{\beta}=\hat{\beta}(t)$. Then, set $\hat{b}(t)=\hat{\alpha}+\hat{\beta} t$ as the approximation of $b(t)$. Alternatively, fit the estimates

and set $\hat{b}(t)=\sqrt{t} \hat{z}$.

$$
\hat{z}=\frac{\hat{\alpha}+\hat{\beta} t}{\sqrt{t}}, \quad \hat{y}=\frac{\hat{\alpha}-\hat{\beta} t}{\sqrt{t}},
$$

This method appears to require the solution of two equations in two unknowns. However, it can be simplified. We aim to solve

$$
(\hat{z}+\hat{y}) \phi(\hat{z})=2 t f(t), \quad \frac{\phi(\hat{z})}{\phi(\hat{y})} \bar{\Phi}(\hat{y})+\bar{\Phi}(\hat{z})=F(t) .
$$


By the first equation,

$$
\hat{y}=\frac{2 t f(t)}{\phi(\hat{z})}-\hat{z}
$$

Call this expression $\hat{y}(\hat{z})$, and substitute it into the second equation:

$$
\frac{\phi(\hat{z})}{\phi(\hat{y}(\hat{z}))} \bar{\Phi}(\hat{y}(\hat{z}))+\bar{\Phi}(\hat{z})=F(t)
$$

This is one equation in the one unknown $\hat{z}$. Upon solving it, set $\hat{b}(t)=\sqrt{t} \hat{z}$. This is our approximation.

Let us show that (1) has a unique solution. Define

$$
\bar{P}(\hat{y}, \hat{z})=\frac{\phi(\hat{z})}{\phi(\hat{y})} \bar{\Phi}(\hat{y})+\bar{\Phi}(\hat{z}), \quad \bar{\Lambda}(\hat{z})=\bar{P}(\hat{y}(\hat{z}), \hat{z}) .
$$

So, $\bar{\Lambda}(\hat{z})$ is the left-hand side of (1). We will need the following integrals:

$$
\begin{aligned}
& \Phi_{1}(x)=\int_{x}^{\infty} \bar{\Phi}(w) \mathrm{d} w=-x \bar{\Phi}(x)+\phi(x), \\
& \Phi_{2}(x)=\int_{x}^{\infty} \Phi_{1}(w) \mathrm{d} w=\frac{1}{2}\left[\left(x^{2}+1\right) \bar{\Phi}(x)-x \phi(x)\right] .
\end{aligned}
$$

Both are everywhere positive.

Lemma 1. We have

$$
\bar{\Lambda}^{\prime}(\hat{z})=-\frac{\phi(\hat{z})}{\phi(\hat{y})}(\hat{y}+\hat{z})\left[\Phi_{1}(\hat{y}) \hat{z}+\bar{\Phi}(\hat{y})\right]
$$

Proof. We have

$$
\begin{aligned}
\frac{\partial \bar{P}}{\partial \hat{z}} & =\frac{\bar{\Phi}(\hat{y})}{\phi(\hat{y})}[-\hat{z} \phi(\hat{z})]-\phi(\hat{z})=-\phi(\hat{z})\left(\frac{\bar{\Phi}(\hat{y})}{\phi(\hat{y})} \hat{z}+1\right) \\
\frac{\partial \bar{P}}{\partial \hat{y}} & =\phi(\hat{z}) \frac{-\phi(\hat{y}) \phi(\hat{y})-\bar{\Phi}(\hat{y})[-\hat{y} \phi(\hat{y})]}{[\phi(\hat{y})]^{2}} \\
& =-\phi(\hat{z}) \frac{-\hat{y} \bar{\Phi}(\hat{y})+\phi(\hat{y})}{\phi(\hat{y})} \\
& =-\phi(\hat{z}) \frac{\Phi_{1}(\hat{y})}{\phi(\hat{y})}, \\
\hat{y}^{\prime}(\hat{z}) & =-\frac{2 t f(t)[-\hat{z} \phi(\hat{z})]}{[\phi(\hat{z})]^{2}}-1=\frac{2 t f(t) \hat{z}}{\phi(\hat{z})}-1=\hat{z}(\hat{y}+\hat{z})-1 .
\end{aligned}
$$

So,

$$
\begin{aligned}
\bar{\Lambda}^{\prime}(\hat{z}) & =\frac{\partial \bar{P}}{\partial \hat{y}} \hat{y}^{\prime}+\frac{\partial \bar{P}}{\partial \hat{z}} \\
& =-\phi(\hat{z}) \frac{\Phi_{1}(\hat{y})}{\phi(\hat{y})}[\hat{z}(\hat{y}+\hat{z})-1]-\phi(\hat{z})\left(\frac{\bar{\Phi}(\hat{y})}{\phi(\hat{y})} \hat{z}+1\right) \\
& =-\frac{\phi(\hat{z})}{\phi(\hat{y})}\left\{\Phi_{1}(\hat{y})[\hat{z}(\hat{y}+\hat{z})-1]+\bar{\Phi}(\hat{y}) \hat{z}+\phi(\hat{y})\right\} \\
& =-\frac{\phi(\hat{z})}{\phi(\hat{y})}\left\{\Phi_{1}(\hat{y})[\hat{z}(\hat{y}+\hat{z})]+[\hat{y} \bar{\Phi}(\hat{y})-\phi(\hat{y})]+\bar{\Phi}(\hat{y}) \hat{z}+\phi(\hat{y})\right\}
\end{aligned}
$$




$$
\begin{aligned}
& =-\frac{\phi(\hat{z})}{\phi(\hat{y})}\left\{\Phi_{1}(\hat{y})[\hat{z}(\hat{y}+\hat{z})]+\bar{\Phi}(\hat{y})(\hat{y}+\hat{z})\right\} \\
& =-\frac{\phi(\hat{z})}{\phi(\hat{y})}(\hat{y}+\hat{z})\left[\Phi_{1}(\hat{y}) \hat{z}+\bar{\Phi}(\hat{y})\right] .
\end{aligned}
$$

Proposition 1. For given $t>0, f(t)>0$, and $0<F(t)<1$, (1) has a unique solution.

Proof. We will use the fact that, for large $x, \bar{\Phi}(x) / \phi(x) \sim 1 / x$.

We have $\hat{y}+\hat{z}>0$ and $\Phi_{1}(\hat{y})>0$, so

$$
\hat{z} \Phi_{1}(\hat{y})+\bar{\Phi}(\hat{y})>-\hat{y} \Phi_{1}(\hat{y})+\bar{\Phi}(\hat{y})=2 \Phi_{2}(\hat{y})>0 .
$$

Consequently, using Lemma $1, \bar{\Lambda}^{\prime}(\hat{z})<0$. Thus, if there is a solution, it is unique.

Moreover, as $\hat{z} \rightarrow \infty, 1 / \phi(\hat{z})$ grows much faster than $\hat{z}$, so $\hat{y} \rightarrow \infty$. Therefore, $\bar{\Phi}(\hat{y}) / \phi(\hat{y})$ $\sim 1 / \hat{y}$, so

$$
\bar{\Lambda}(\hat{z}) \sim \frac{\phi(\hat{z})}{\hat{y}}+\bar{\Phi}(\hat{z}) \rightarrow 0 .
$$

As $\hat{z} \rightarrow-\infty$, again $\hat{y} \rightarrow \infty$. Therefore, $\bar{\Phi}(\hat{y}) / \phi(\hat{y}) \sim 1 / \hat{y}$, so

$$
\bar{\Lambda}(\hat{z}) \sim \frac{\phi(\hat{z})}{\hat{y}}+\bar{\Phi}(\hat{z}) \rightarrow 1 .
$$

Thus, the range of $\bar{\Lambda}$ is the entire interval $(0,1)$, so (1) does have a solution.

The proof indicates that $\bar{\Lambda}$ has all the properties of a complimentary distribution function. Thus, solving (1) can be interpreted as solving a version of the newsvendor problem. This interpretation is meaningful in the context of the inventory model of [12].

It is worth mentioning a couple of even simpler estimates. The following is a lower bound on $b$ :

$$
b^{-}(t)=\sqrt{t} \bar{\Phi}^{-1}(F(t)) .
$$

(This was shown independently by Cheng et al. [2] and Song and Zipkin [12].) Since (1) implies that $\bar{\Phi}(\hat{z})<F(t)$, we have $\hat{b}(t)>b^{-}(t)$. Another simple estimate is

$$
b^{+}(t)=\sqrt{t} \bar{\Phi}^{-1}\left(\frac{1}{2} F(t)\right) .
$$

Under a certain condition (essentially, when $b^{+}(t)$ itself is nonincreasing — see [2]), this is an upper bound on $b$. (None of the examples below has this property.) It exactly equals $b$ in the special case where $b$ is constant, that is, where $b(t)=\alpha+\beta t$ with $\beta=0$. As we will see, these two estimates are not reliably accurate.

Here are a few remarks about the numerical issues. First, the evaluation of the inverse Gaussian distribution can be numerically challenging when $y$ is very large, for then $\phi(z) / \phi(y)$ becomes large, while $\bar{\Phi}(y)$ is small. In this case, we can use the fact that $\bar{\Phi}(y) / \phi(y) \sim 1 / y$, so

$$
F_{(\alpha, \beta)}(t) \sim \frac{\phi(z)}{y}+\bar{\Phi}(z)
$$

In solving (1), therefore, when we encounter a large $\hat{y}(\hat{z})$, we can adjust the equation to read

$$
\frac{\phi(\hat{z})}{\hat{y}(\hat{z})}+\bar{\Phi}(\hat{z})=F(t) .
$$


Also, we have found it useful to transform (1) as follows:

$$
\Phi^{-1}\left(\frac{\phi(\hat{z})}{\phi(\hat{y}(\hat{z}))} \bar{\Phi}(\hat{y}(\hat{z}))+\bar{\Phi}(\hat{z})\right)=\Phi^{-1}(F(t)) .
$$

This, in our experience, speeds up the calculation.

Incidentally, if $f(t)=0$ on some interval then, by the definition of $\tau$, the boundary $b(t)$ does not exist on that interval. We might say that $b(t)=\infty$. For example, if $f(t)=0$ and $F(t)=1$ for an interval $(T, \infty)$, the distribution is concentrated on $[0, T]$, and the boundary $b(t)$ does not exist for $t>T$. We should and usually can identify such intervals in advance. The method above applies to the other intervals.

\section{Method of images}

Some of the examples we test below are constructed by the method of images (see [4] and [8]). This method enables us to synthesize a boundary $b$ and the corresponding distribution $F$ with fairly simple calculations. The approach begins with a positive, $\sigma$-finite measure $A$ on the positive real numbers. Define

$$
q(t, x)=\frac{1}{\sqrt{t}} \phi\left(\frac{x}{\sqrt{t}}\right)-\int_{0}^{\infty} \frac{1}{\sqrt{t}} \phi\left(\frac{\xi-x}{\sqrt{t}}\right) A(\mathrm{~d} \xi),
$$

and, for each $t>0$, set $b(t)$ to the value of $x$ which solves the equation

$$
q(t, x)=0
$$

There is a unique solution. Then,

$$
\begin{aligned}
& f(t)=\frac{1}{2 t^{3 / 2}} \int_{0}^{\infty} \xi \phi\left(\frac{\xi-b(t)}{\sqrt{t}}\right) A(\mathrm{~d} \xi) \\
& F(t)=\bar{\Phi}\left(\frac{b(t)}{\sqrt{t}}\right)+\int_{0}^{\infty} \bar{\Phi}\left(\frac{\xi-b(t)}{\sqrt{t}}\right) A(\mathrm{~d} \xi) .
\end{aligned}
$$

The boundary $b(t)$ is always smooth and concave.

When the measure $A$ is concentrated on a single positive point, we recover the linear boundaries discussed above. Suppose that $A$ is concentrated on a finite number $J$ of positive, equally spaced points, say at $j \xi$ for some $\xi>0, j=1, \ldots, J$. Denote their weights by $a_{j} \geq 0$. Also, set $a_{0}=-1$. As discussed in [4] and [12], in this case $q$ becomes

$$
q(t, x)=\frac{1}{\sqrt{t}} \phi\left(\frac{x}{\sqrt{t}}\right)-\sum_{j=1}^{J} a_{j} \frac{1}{\sqrt{t}} \phi\left(\frac{j \xi-x}{\sqrt{t}}\right),
$$

and (2) reduces to the polynomial equation

$$
\sum_{j=0}^{J} \tilde{a}_{j}(t) y^{j}=0
$$

where $\tilde{a}_{j}(t)=a_{j} \exp \left(-(j \xi)^{2} / 2 t\right)$ and $y=\exp (\xi x / t)$. In particular, $\tilde{a}_{0}(t)=a_{0}=-1, t>0$. By the definition of $y$, only a positive root of (3) corresponds to a solution of (2), and, in fact, 
there is a unique positive root. Given the root $y$, we can set $b(t)=(t / \xi) \ln (y)$. Assuming that $a_{1}>0$, it turns out that $b(0)=\frac{1}{2} \xi$. Then,

$$
\begin{aligned}
& f(t)=\frac{\xi}{2 t^{3 / 2}} \sum_{j=1}^{J} j a_{j} \phi\left(\frac{j \xi-b(t)}{\sqrt{t}}\right), \\
& F(t)=\bar{\Phi}\left(\frac{b(t)}{\sqrt{t}}\right)+\sum_{j=1}^{J} a_{j} \bar{\Phi}\left(\frac{j \xi-b(t)}{\sqrt{t}}\right) .
\end{aligned}
$$

For large $t$, the boundary $b$ is asymptotically linear, and its limiting slope, say $\beta$, has the same sign as $1-\sum_{j=1}^{J} a_{j}$. The large- $t$ behavior of $F$ depends on $\beta$ in the same way as an inverse Gaussian distribution, as discussed above.

Next, consider the measure $A(\mathrm{~d} \xi)=\gamma \exp (\kappa \xi) \mathrm{d} \xi$, where $\gamma>0$, but $\kappa$ can be any real number. (The $\kappa=0$ case was analyzed in [8, Example 7, p. 30], and Song and Zipkin [12] examined the $\kappa<0$ case.) We can show that

$$
q(t, x)=\frac{1}{\sqrt{t}} \phi\left(\frac{x}{\sqrt{t}}\right)-\gamma \exp \left(\frac{1}{2} \kappa^{2} t+\kappa x\right) \Phi\left(\frac{\kappa t+x}{\sqrt{t}}\right)
$$

so (2) is equivalent to

$$
0=\exp \left(-\frac{1}{2} \kappa^{2} t-\kappa x\right) \phi\left(\frac{x}{\sqrt{t}}\right)-\gamma \sqrt{t} \Phi\left(\frac{\kappa t+x}{\sqrt{t}}\right)=\phi\left(\frac{\kappa t+x}{\sqrt{t}}\right)-\gamma \sqrt{t} \Phi\left(\frac{\kappa t+x}{\sqrt{t}}\right) .
$$

Defining

the equation reduces to

$$
v=\frac{\kappa t+x}{\sqrt{t}}
$$

$$
\frac{\phi(v)}{\Phi(v)}=\gamma \sqrt{t}
$$

Given the solution $v=v(t)$, set $b(t)=-\kappa t+\sqrt{t} v(t)$. It is not hard to show that, as $t \rightarrow 0, v(t) \rightarrow \infty$, but $b(t) \sim \sqrt{-t \ln \left(\gamma^{2} 2 \pi t\right)} \rightarrow 0$. As $t \rightarrow \infty, v(t) \sim-\gamma \sqrt{t}$, so $b(t)$ is asymptotically linear with slope $-(\kappa+\gamma)$. Also, the limiting intercept is $1 / \gamma$.

Then,

$$
F(t)=\bar{\Phi}\left(\frac{b(t)}{\sqrt{t}}\right)+\int_{0}^{\infty} \gamma \exp (\kappa \xi) \bar{\Phi}\left(\frac{\xi-b(t)}{\sqrt{t}}\right) \mathrm{d} \xi
$$

For $\kappa \neq 0$, this becomes

$$
\begin{aligned}
F(t) & =1-\frac{1}{\kappa}\left[(\kappa+\gamma) \Phi\left(\frac{b(t)}{\sqrt{t}}\right)-\frac{1}{\sqrt{t}} \phi\left(\frac{b(t)}{\sqrt{t}}\right)\right] \\
& =1-\frac{1}{\kappa}\left[(\kappa+\gamma) \Phi(v(t)-\kappa \sqrt{t})-\frac{1}{\sqrt{t}} \phi(v(t)-\kappa \sqrt{t})\right] .
\end{aligned}
$$

For $\kappa=0$,

$$
F(t)=1+\left(\gamma \sqrt{t}-\frac{1}{\gamma \sqrt{t}}+v(t)\right) \phi(v(t)) .
$$

In both cases, the density can be written as

$$
f(t)=\frac{1}{2 t}(v(t)+\gamma \sqrt{t}) \phi(v(t)-\kappa \sqrt{t}) .
$$

Again, the large- $t$ behavior of $F$ depends on the asymptotic slope of $b$, namely $-(\kappa+\gamma)$. 


\section{Illustrations}

Let us try the method on some examples.

\subsection{Method of images}

First, we consider several examples constructed by the method of images.

4.1.1. Two points. Figure 1 shows a case with $J=2, \xi=1$, and $a_{j}=\frac{1}{2}, j=1,2$. It displays the exact solution $b$, along with the approximation $\hat{b}$, the lower bound $b^{-}$, and the other simple estimate $b^{+}$. It also displays the distribution $F$, measured on the scale on the right for reference. Evidently, the approximation is quite close to the true boundary. It slightly underestimates the exact solution. The simpler estimates $b^{-}$and $b^{+}$are not accurate.

Figure 2 displays the deviations, both absolute, $|b-\hat{b}|$, and relative, $|b-\hat{b}| / b$, over a larger time scale. They continue to grow as $t$ increases, but very gently.

In this case $b$ has asymptotic slope $\beta=0$ (because $\sum_{j=1}^{J} a_{j}=1$ ). We have also tried cases with positive and negative asymptotic slopes (having smaller and larger $a_{j}$, respectively), and the results are similar. The approximation appears to have the correct asymptotic slope. Likewise, the results are similar for cases with more points, i.e. $J>2$.

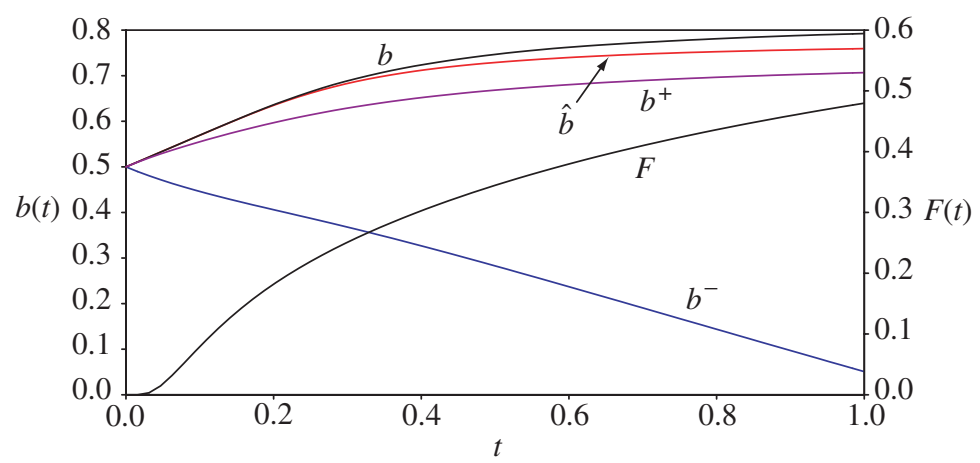

Figure 1: Two-point approximation with $\xi=1, a_{1}=0.5$, and $a_{2}=0.5$.

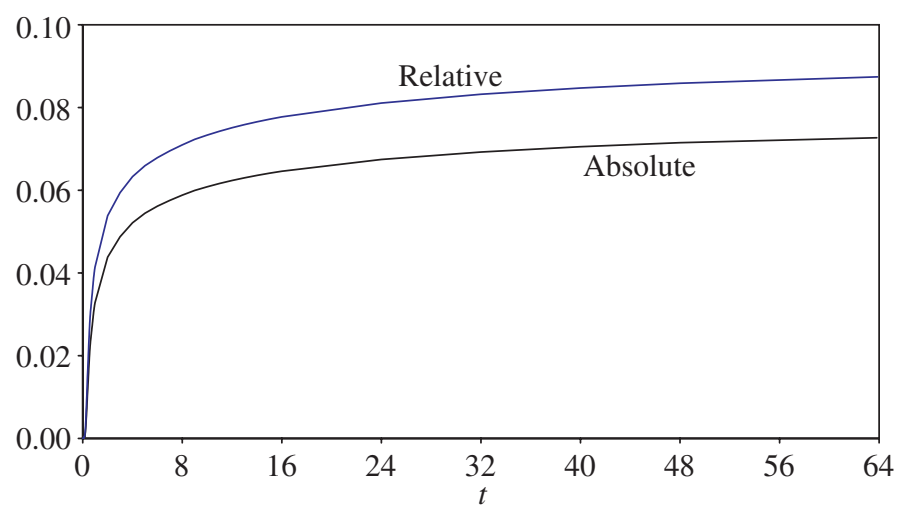

FIgURE 2: The deviations of the two-point approximation over a larger time scale. 
4.1.2. Mixed signs. As pointed out by Daniels [5] and Lo et al. [9], the measure $A$ need not be positive everywhere. For a discrete measure, some of the $a_{j}$ may be negative. Here, $b(t)$ need not be concave or smooth.

Consider the rather remarkable curve in Figure 3. (What causes such strange behavior? In this case, at $t=\bar{t}=1 / \ln (6)$, all three roots of the cubic equation (3) coincide. For $t<\bar{t}$, there are three distinct positive roots, while, for $t>\bar{t}$, there is only one.) The boundary $b$ is continuous, but it is certainly not smooth. As $t$ approaches $\bar{t}$ from below, its slope becomes infinite. The density $f$ is continuous, but $f(\bar{t})=0$, and the shape of $F$ clearly indicates that $f$ is bimodal. The approximation $\hat{b}$ fails spectacularly near $\bar{t}$. In fact, it goes to $\infty$ as $t$ approaches $\bar{t}$ from below or above.

Figure 4 shows a less extreme curve, resulting from a slightly larger (less negative) $a_{2}$. (In this case, two roots coincide at a certain value $\bar{t}$.) Here too, $f$ is bimodal. The approximation is still poor in some places, although not as bad as in the previous case.

In both cases, for larger $t$, both $b(t)$ and $\hat{b}(t)$ continue to decrease almost linearly. There is no more strange behavior, and the error of the approximation does not get any worse.

Evidently, these examples push the approximation beyond its limits as a reliable numerical device. We suspect that such cases may prove challenging for other methods for the FPT and the IFPT problems. As far as the authors are aware, none of the available methods has been tested on such cases.

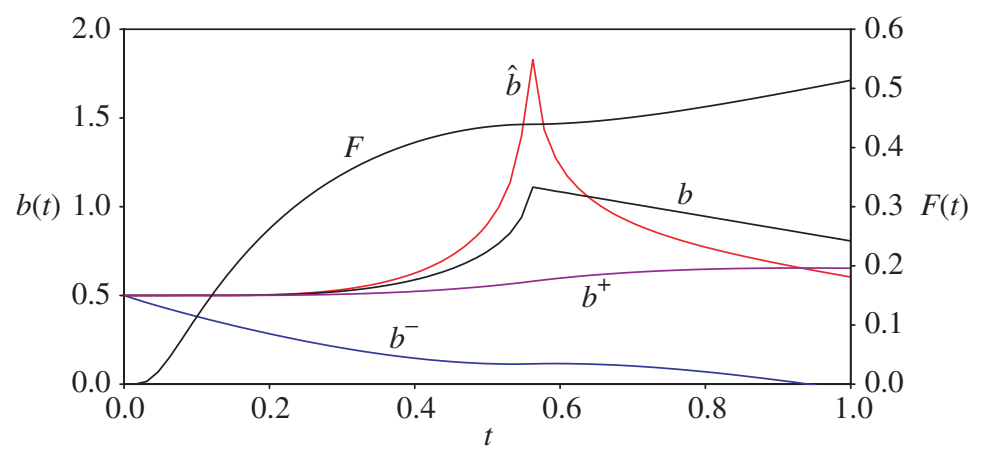

FIGURE 3: Three-point approximation with $\xi=1, a_{1}=1, a_{2}=-2$, and $a_{3}=8$.

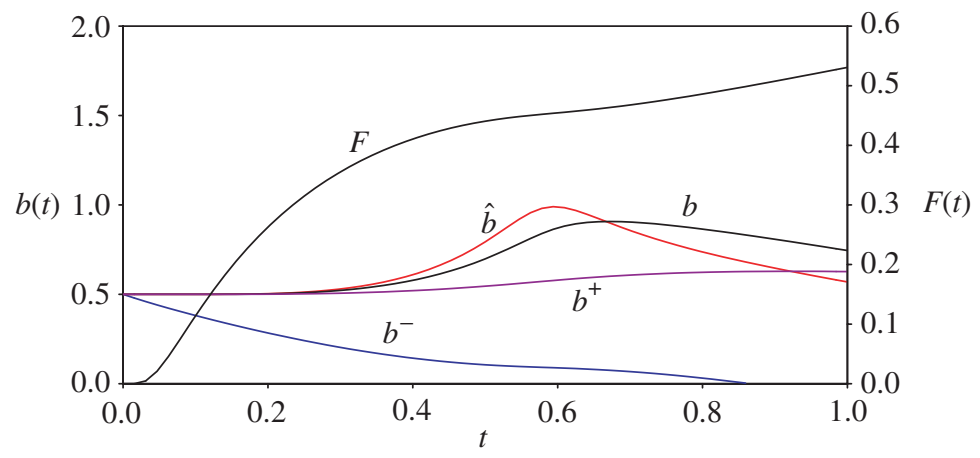

FIGURE 4: Three-point approximation with $\xi=1, a_{1}=1, a_{2}=-1.85$, and $a_{3}=8$. 


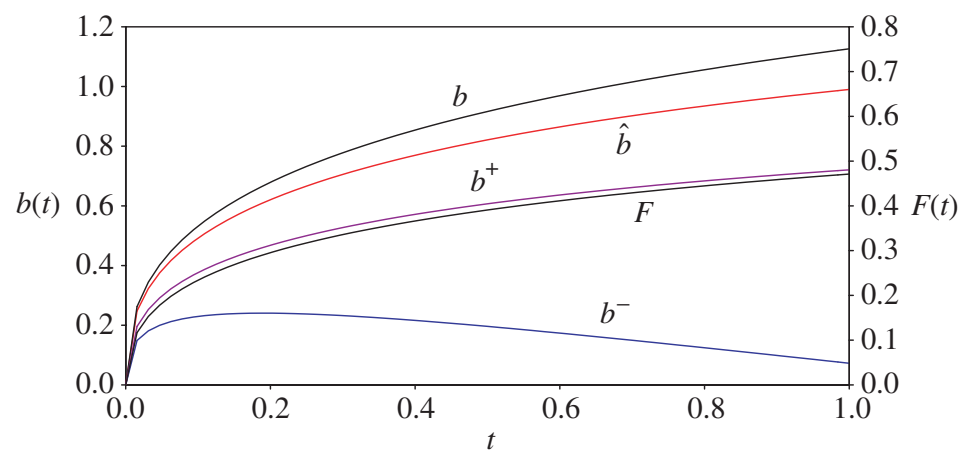

FIGURE 5: Exponential measure with $\gamma=0.4$ and $\kappa=-0.4$.

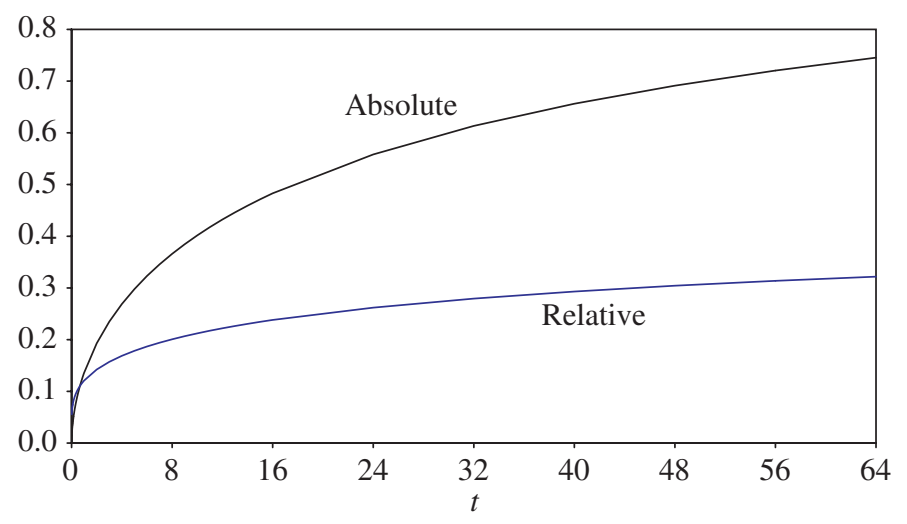

FIgURE 6: The deviations of the exponential measure over a larger time scale.

4.1.3. Exponential measure. Figure 5 shows the case of the exponential measure $A(\mathrm{~d} \xi)=$ $\gamma \exp (\kappa \xi) \mathrm{d} \xi$ with $\gamma=0.4$ and $\kappa=-0.4$. Here, $\hat{b}$ mimics the shape of $b$, though not as precisely as in Figure 1. Figure 6 shows what happens for larger $t$. The errors grow as $t$ increases, but not too much. Again, we have also tried cases with nonzero asymptotic slopes, and the results are similar.

\subsection{Distributions with finite support}

We now consider some distributions with finite support, specifically on $(0,1)$. These cases are interesting in themselves, and they are important in the inventory models of [12]. None of these has an explicit solution, to the authors' knowledge, and so we use the second (Volterra equation) method of [14] to estimate $b$.

Figures 7-8 display the results for two cases. The first shows the uniform distribution on $(0,1)$, and the second shows a quadratic $F$. In both cases, the boundary $b(t)$ plunges to $-\infty$ as $t$ approaches 1 . This is as it should be, considering the meaning of $\tau$. The approximation $\hat{b}$ is quite accurate. Interestingly, in contrast to the previous cases, the lower bound $b^{-}$is not too far off either. 


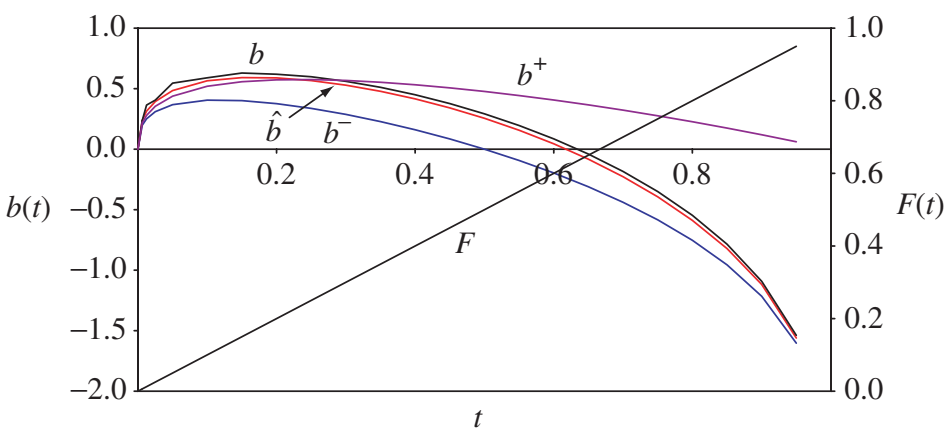

FIGURE 7: Distribution with uniform finite support.

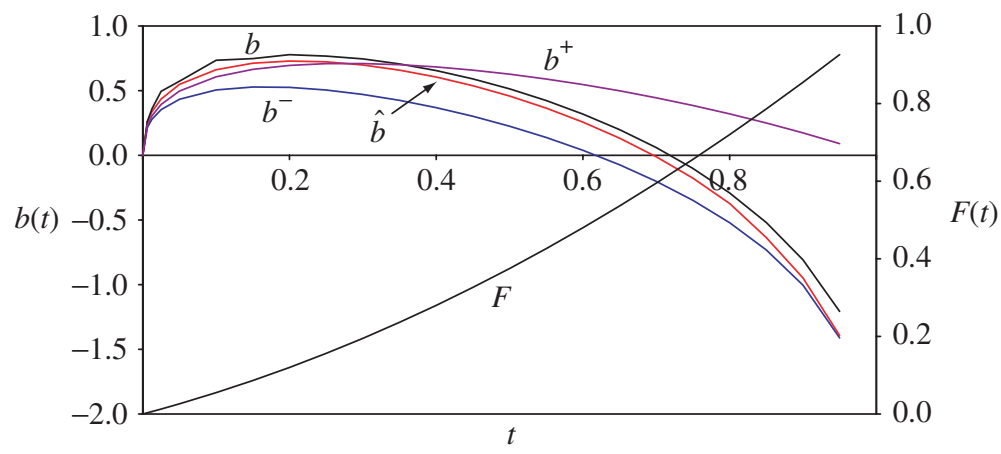

FIGURE 8: Distribution with quadratic, $F(t)=0.5 t(1+t)$, finite support.

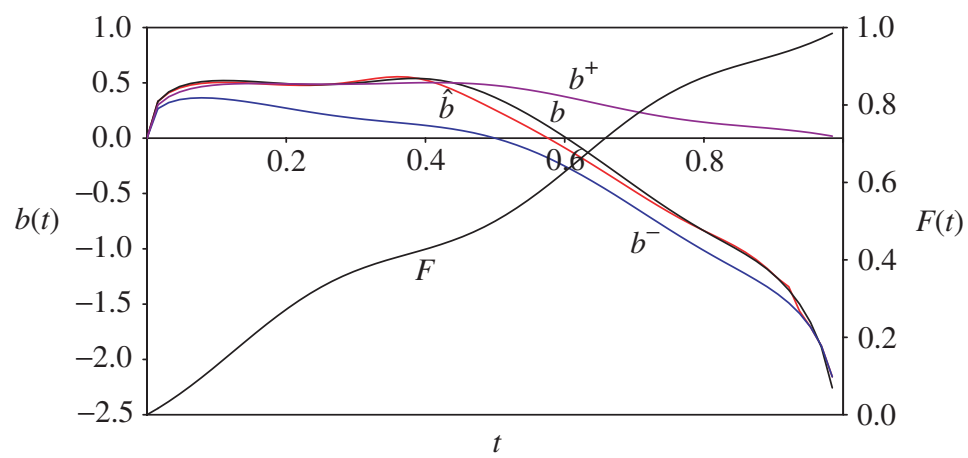

FIGURE 9: Distribution with finite support of the form (4), where $n=2$ and $\gamma=0.5$.

Next, we consider densities of form

$$
f(t)=1+\gamma \sin (2 \pi n t), \quad F(t)=t+\frac{\gamma}{2 \pi n}[1-\cos (2 \pi n t)],
$$

where $0<\gamma<1$ and $n$ is a positive integer. Figures 9-10 show the results for two cases, both 


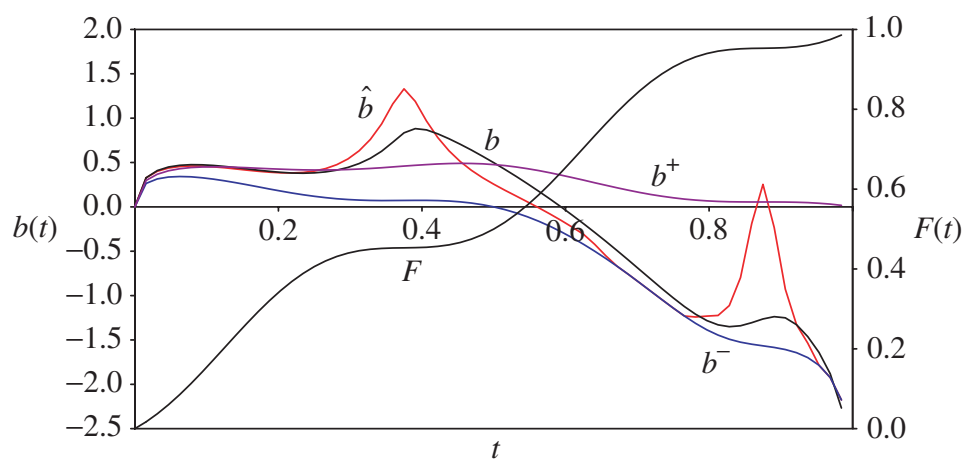

FIGURE 10: Distribution with finite support of the form (4), where $n=2$ and $\gamma=0.98$.

with $n=2$. The first has $\gamma=0.5$. The approximation is quite accurate. The second case has $\gamma=0.98$. Here, $\hat{b}$ is not accurate. The problem seems to be that $f(t)$ is nearly 0 between its peaks, as in Figures 3-4 above.

\section{Discussion}

We have seen that the proposed approximation works quite well in several cases, though not all. It performs best when the density $f$ is unimodal. It runs into trouble when $f$ is multimodal, specifically when $f$ approaches 0 between modes. Such behavior is very different from that of an inverse Gaussian density, on which the method is based. It would be interesting to conduct a serious error analysis of the method.

Of course, our method can be used to provide a starting solution for recursive algorithms like those of [12] and [14]. Can the method itself be improved? Durbin [6] developed a series expansion of the exact solution of the FPT problem, whose first term is the tangent approximation. Perhaps something similar can be done here. It would be especially valuable to develop methods that are robust in dealing with near-flat distributions and other anomalies, like those of Figures 3, 4 and 10.

\section{Acknowledgement}

Song's research was supported in part by the National Natural Science Foundation of China, grant number 70731003 .

\section{References}

[1] Abundo, M. (2006). Limit at zero of the first-passage time density and the inverse problem for one-dimensional diffusions. Stoch. Anal. Appl. 24, 1119-1145.

[2] Cheng, L., Chen, X., Chadam, J. and Saunders, D. (2007). Analysis of an inverse first passage problem from risk management. SIAM J. Math. Anal. 38, 845-873.

[3] Daniels, H. E. (1974). The maximum size of a closed epidemic. Adv. Appl. Prob. 6, 607-621.

[4] Daniels, H. E. (1982). Sequential tests constructed from images. Ann. Statist. 10, 394-400.

[5] Daniels, H. E. (1996). Approximating the first crossing-time density for a curved boundary. Bernoulli 2, 133143.

[6] Durbin, R. (1992). The first-passage density of the Brownian motion process to a curved boundary. J. Appl. Prob. 29, 291-304. 
[7] Jaimungal, S., Kreinin, A. And Valov, A. (2009). Integral equations and the first passage time of Brownian motion. Working paper, University of Toronto.

[8] Lerche, H. R. (1986). Boundary Crossing of Brownian Motion. Springer, Berlin.

[9] Lo, V. S. F., Roberts, G. O. And Daniels, H. E. (2002). Inverse method of images. Bernoulli 8, 53-80.

[10] Peskir, G. (2002). On integral equations arising in the first-passage problem for Brownian motion. J. Integral Equations Appl. 14, 397-423.

[11] Roberts, G. O. ANd Shortland, C. F. (1995). The hazard rate tangent approximation for boundary hitting times. Ann. Appl. Prob. 5, 446-460.

[12] Song, J. And Zipkin, P. (2006). Supply streams. Working paper, Duke University.

[13] Strassen, V. (1967). Almost sure behavior of sums of independent random variables and martingales. In Proc. 5th Berkeley Symp. Math. Statist. Prob., Vol. II, University of California Press, Berkeley, CA, pp. 315-343.

[14] Zucca, C. ANd Sacerdote, L. (2009). On the inverse first-passage-time problem for a Wiener process. Ann. Appl. Prob. 19, 1319-1346. 